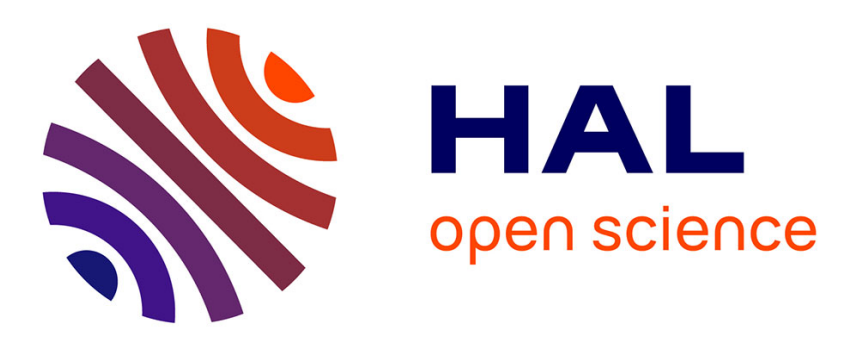

\title{
De la description définie au nom propre de personne: sur un apport possible de l'anthroponymie historique à la théorie du nom propre modifié \\ Eva Buchi, Aude Wirth
}

\section{- To cite this version:}

Eva Buchi, Aude Wirth. De la description définie au nom propre de personne: sur un apport possible de l'anthroponymie historique à la théorie du nom propre modifié. Langue française, 2005, 146, pp.23-38. halshs-00005015

\section{HAL Id: halshs-00005015 \\ https://shs.hal.science/halshs-00005015}

Submitted on 17 Oct 2005

HAL is a multi-disciplinary open access archive for the deposit and dissemination of scientific research documents, whether they are published or not. The documents may come from teaching and research institutions in France or abroad, or from public or private research centers.
L'archive ouverte pluridisciplinaire HAL, est destinée au dépôt et à la diffusion de documents scientifiques de niveau recherche, publiés ou non, émanant des établissements d'enseignement et de recherche français ou étrangers, des laboratoires publics ou privés. 
Éva Buchi \& Aude Wirth

UMR CNRS 7118 ATILF

Université Nancy II

\section{De la description définie au nom propre de personne : sur un apport possible de l'anthroponymie historique à la théorie du nom propre modifié}

\section{Objectif, méthode, base documentaire}

1.1. Cette contribution à la réflexion sur la modification du nom propre entend renouer le dialogue entre onomastique historique et théorie du nom propre, deux disciplines dont le divorce est déjà ancien ${ }^{1}$, mais dont les résultats respectifs, pensons-nous, sont appelés à s'enrichir mutuellement.

1.2. Le corpus sur lequel nous nous appuierons est constitué de la partie consacrée à la France d'oïl de la base de données anthroponymiques élaborée dans le cadre du projet PatRom (Patronymica Romanica. Dictionnaire historique de l'anthroponymie romane). Ce projet réunit une quinzaine d'équipes de chercheurs d'expression romane, du Portugal à la Roumanie $^{2}$; il a pour but l'étymologisation du noyau commun du stock anthroponymique d'origine lexicale des différents pays européens de langue romane ${ }^{3}$. Pour le domaine qui nous intéresse, la base de données PatRom regroupe 172.464 occurrences tirées de 184 sources historiques (cartulaires, rôles de taille, obituaires, dénombrements, etc.) du $11^{\mathrm{e}}$ au $18^{\mathrm{e}}$ siècle ${ }^{4}$.

Ce corpus, qui a été établi selon des critères philologiques solides, permet de saisir les noms propres de personnes français dans leur usage réel à travers les siècles. En dépit de sa richesse et de sa fiabilité, cette base est encore largement sous-exploitée : pour l'heure, elle n'a été utilisée que pour l'élaboration des 290 articles (ALACER, ALAMANNUS, ALBUS, AMĀ-

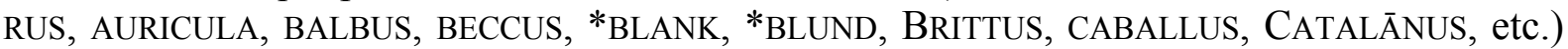
du dictionnaire PatRom en cours de publication. Notre recherche en constitue une exploitation d'un autre ordre, syntaxique plutôt que lexical. Le recours à ce vaste corpus fiable s'imposait d'autant plus que la réflexion des théoriciens du nom propre s'appuie bien souvent sur une base documentaire assez restreinte et en général limitée à l'époque contemporaine.

1.3. Concrètement, nous nous proposons de réfléchir au statut de l'article défini non agglutiné sur lequel s'ouvrent certains noms propres de personne médiévaux. Retenant ce seul critère comme indice de modification, nous nous rangeons donc résolument du côté des théoriciens du nom propre qui privilégient l'approche syntaxique, au détriment de l'approche sémantique, qui se prête moins bien à l'analyse d'états de langue anciens (cf. Noailly 1999, 109-111 pour une discussion des deux points de vue).

Voici quelques exemples relevés dans les sources patromiennes ${ }^{5}$ :

\footnotetext{
${ }^{1}$ Cf. Chambon 1988, 177 : «On signale ici ce numéro de Langages [...]. Les onomasticiens auxquels il aurait échappé s'y reporteront avec profit. Non seulement parce que les cinq contributions [...] illustrent — partiellement - la diversité des approches actuelles du nom propre (en logique, en philosophie du langage, en psycholinguistique, en anthropologie, notamment), mais encore parce qu'ils verront comment leur discipline est perçue de l'extérieur [5, 103, 104, 105]. L'absence de représentation de l'onomastique en tant que discipline historico-philologique [...] est symptomatique. L'onomastique française serait-elle en passe de se laisser évincer — sans même, peut-être, s'en apercevoir — de son propre domaine ?»

2 Cf. Kremer 1997 et Cano/Germain/Kremer 2004-; cf. aussi le site Internet du projet (http://patrom.fltr.ucl.ac.be/). Pour l'approche méthodologique, cf. Chambon 1996; 1998 ; Buchi 2001 ; Wirth 2003.

${ }^{3}$ Cf. aussi le projet du GREHAM (Groupe de Recherche Européen sur l'Histoire de l'Anthroponymie Médiévale), dirigé par la médiéviste Monique Bourin (Bourin 1990-2002), dont les objectifs sont complémentaires.

${ }^{4}$ On a mis à part les 130.969 occurrences de la base PatRom pour la Belgique romane, qu'il serait intéressant d'exploiter dans une publication ultérieure. - Nos remerciements s'adressent à Jean Germain et à Boris Maroutaeff de l'Université de Louvain-la-Neuve pour la communication de ce chiffre.

${ }^{5}$ Dans un souci de gain de place, nous avons renoncé à indiquer les références bibliographiques des occurrences citées. Le lecteur trouvera la bibliographie des sources PatRom sur le site Internet du projet (cf. note 2).
} 
li Berart (Niort $13^{\mathrm{e}} \mathrm{s}$.)

la Barbiere (Pontoise 1287)

le Clerc poessonnier (Blois 1389)

li sires Forquez de Jurue (Metz 1251)

li dicte dame Marie Maqueriele (Cysoing 1393)

Le regroupement de ces cas de figure pourra paraître étonnant. En effet, les différences entre les cinq occurrences citées sont manifestes, qu'elles concernent leur structure interne, la classe étymologique des éléments qui les composent ou encore le statut de l'article initial. Ce qui les rapproche, en revanche, c'est qu'ils répondent tous à la définition de ce que nous appellerons une chaîne anthroponymique : un syntagme nominal comportant au minimum un nom propre de personne ${ }^{6}$ (éventuellement accompagné d'une expansion), dont le référent est un être humain et qui forme une unité dans un ensemble discursif. Typiquement, dans les documents dont le dépouillement est à l'origine de la base de données PatRom, cet ensemble discursif est une liste de noms de personnes, et les chaînes anthroponymiques forment les unités du paradigme ainsi formé.

1.4. Pour que le lecteur puisse se faire une idée un peu plus concrète de la nature de ce type de documents, nous en présenterons ici un exemple. Il s'agit d'un extrait du rôle d'imposition de 1425 d'une localité de Lorraine (Châtel-sur-Moselle [Vosges]), que l'une d'entre nous a eu l'occasion d'éditer (cf. Wirth 2002, vol. 2, 3) :

C'est le paipier de l'ayde faite a monseigneur
en sa terre de Lorrenne pour le
fait de sa guerre contre l'evesque de
$\begin{array}{ll}\text { Basle et cl ou mois de decembre } \\ \text { l'an mil .iiiic xxv. - Cet premiers } \\ \text { Chastel la ville } & \text {.iii. florins } \\ \text { Xaievin } & \text {.ii. florins } \\ \text { Rolin } & \text {.vi. gros } \\ \text { la femme Ferriot } & \text {.iii. florins } \\ \text { Hesselewat } & \text {.ii. florins } \\ \text { Mengin Pourcel } & \text {.viii. florins } \\ \text { Jehan Werrey } & \text {.vii. gros } \\ \text { la femme Wauterin } & \text {.iii. florins } \\ \text { Girart Bouart } & \text {.iii. florins } \\ \text { Jaiquot tixerant } & \text {.viii. florins } \\ \text { le berbier Estienne } & \end{array}$

La structure du texte est comparable à celle d'un livre de comptes : la colonne de gauche fait la liste les contribuables, tandis que celle de droite précise la somme que ces derniers ont versée. Ce qui frappe, c'est la diversité des dénominations (noms de personnes nus et différents types de chaînes anthroponymiques complexes). Ainsi le berbier Estienne est cité au même niveau que Xaievin ou Rolin : les chaînes anthroponymiques comportant un article entrent dans le même paradigme que des noms de personnes non modifiés ${ }^{7}$.

On pourrait dire que le rapport entre un nom propre de personne (Xaievin, Rolin, Ferriot) et une chaîne anthroponymique (Xaievin, Rolin, la femme Ferriot) est de même nature que celui entre un nom commun et un syntagme nominal ${ }^{8}:$ le basculement de l'anthro-

\footnotetext{
${ }^{6}$ Il va de soi qu'étymologiquement, ce nom propre de personne peut être un nom de personne, mais aussi un lexème, un nom de lieu, voire un énoncé (cas des délocutifs).

${ }^{7}$ Nous avons toutefois exclu de la nomenclature les chaînes anthroponymiques comme le fils Guillaume Torel (Normandie 1340) ou la fame Symon lymagier (Paris 1300), dont la particularité consiste à reposer sur un cas sujet suivi d'un cas régime complément déterminatif, et qui mériteraient d'être analysées dans une publication à part.

${ }^{8} \mathrm{Cf}$. Flaux 1995 pour une proposition de rattachement du nom propre à la classe des syntagmes nominaux.
} 
ponyme à la chaîne anthroponymique équivaut au passage de l'ordre de la langue à celui du discours (même si, en l'occurrence, il s'agit d'un discours d'un type particulier').

1.5. Une dernière précision, dans le but de situer notre propos par rapport aux autres contributions de ce volume. Parmi les trois groupes de noms de personnes modifiés que Kleiber $(1992,186)$ distingue sur la base d'un critère référentiel, les items ici analysés se classent dans la catégorie de "ceux où le référent continue d'être un ou le porteur du nom» : le berbier Estienne renvoie bien au coiffeur appelé Estienne et non pas à un seul aspect de cet Estienne, et encore moins à une entité unie à cet Estienne par une certaine relation. Plus précisément, les chaînes anthroponymiques ici analysées appartiennent à la classe des emplois que K. Jonasson appelle dénominatifs (cf. Jonasson 1994, 182-214) et que G. Kleiber (1981, 385-404) décrit typiquement à l'aide du prédicat de dénomination être appelé $/ N /(x)$.

\section{Résultats}

\section{1. Évaluation quantitative}

Sur un total de 172.464 chaînes anthroponymiques que comporte la base PatRom pour le domaine de la France d'oïl, 1.902 commencent par un article défini, que ce dernier soit $l i$ (797 cas ou 42\%), le (736 cas ou 39\%), la (266 cas ou 14\%), l(') (72 cas ou 4\%), lou (vingtsept cas ou 1\%), lo (trois cas ou moins d' $1 \%$ ), lu ou $l y$ (un seul cas ou moins d'1\% chacun). Ces 1.902 chaînes anthroponymiques, que l'on pourrait appeler modifiées (parce qu'elles s'ouvrent sur un article défini, «modificateur» classique), représentent juste un peu plus de 1\% de l'ensemble des chaînes anthroponymiques de la base de données : il s'agit visiblement d'un phénomène marginal.

Il ne faut toutefois pas oublier qu'au sein des chaînes anthroponymiques de notre corpus, l'article défini a une fréquence plus élevée que celle que nous venons de mettre en évidence : d'une part, on rappellera le cas particulier des cas sujets suivis d'un cas régime complément déterminatif (comme le fils Guillaume Torel ou la fame Symon lymagier, cf. note 7), que nous avons volontairement exclu de notre étude; d'autre part, l'article défini peut apparaître à l'intérieur de la chaîne anthroponymique et ne déterminer qu'un élément la composant: Petrus li Chardoniers (Paris a.1216), Goudefrin lou Bague (Metz a.1279), Mahieu de Claires l'ainsné (Rouen a.1381).

\section{2. Évaluation qualitative}

\subsubsection{Introduction}

Le tableau synoptique ci-dessous intègre trois critères disponibles pour l'évaluation qualitative de nos résultats : l'aspect diachronique, qui, comme on le verra, n'est pas pertinent ici, l'aspect catégoriel (nature du pivot ou élément suivant directement l'article défini) et l'aspect syntaxique (structure interne de la chaîne anthroponymique). Deux autres critères utiles à l'évaluation qualitative seront introduits au fur et à mesure de l'exposé : l'aspect diatopique et l'aspect morphologique, ce dernier ne concernant que les féminins.

\begin{tabular}{|c|c|c|c|c|c|c|c|c|c|c|}
\hline & Total & $11^{\mathrm{e}} \mathrm{s}$. & $12^{\mathrm{e}} \mathrm{s}$ & $13^{\mathrm{e}} \mathrm{s}$ & $14^{\mathrm{e}} \mathrm{s}$ & $15^{\mathrm{e}} \mathrm{s}$ & $16^{\mathrm{e}} \mathrm{s}$ & $17^{\mathrm{e}} \mathrm{s}$ & & $8^{\mathrm{e}} \mathrm{s}$ \\
\hline Article + lexème & 800 & & 2 & 140 & 523 & 99 & 14 & & 6 & 16 \\
\hline Article + lexème $+X$ & 715 & & 1 & 226 & 414 & 43 & 6 & 6 & 2 & 23 \\
\hline Article + titre + lexème $+X$ & 26 & & 1 & 5 & 17 & & & & 1 & 2 \\
\hline Article + titre + nom de personne $+X$ & 207 & & & 114 & 91 & & & & 2 & \\
\hline
\end{tabular}

\footnotetext{
${ }^{9}$ La formulation «production langagière réglée dans laquelle les signes onomastiques tiennent un rôle de premier plan » (Chambon 2004, 105 [ici au sujet du censier interpolé de la charte de fondation du monastère de Sauxillanges]) nous paraît particulièrement heureuse.
} 


\begin{tabular}{|c|c|c|c|c|c|c|c|c|c|}
\hline Article + introducteur + lexème & 9 & & & 5 & & 2 & 1 & & 1 \\
\hline Article + introducteur + lexème $+X$ & 4 & & & 1 & & & & & 3 \\
\hline Article + introducteur + nom de pers. & 2 & & & 1 & 1 & & & & \\
\hline Article + introducteur + nom de pers. $+X$ & 18 & & & 8 & 7 & 2 & 1 & & \\
\hline Article + introducteur + nom de lieu & 2 & & & 1 & 1 & & & & \\
\hline Article + nom de personne & 95 & 1 & 2 & 27 & 43 & 22 & & & \\
\hline Article + nom de personne $+X$ & 22 & & & 12 & 7 & 2 & 1 & & \\
\hline Article + nom de lieu & 2 & & & 1 & & 1 & & & \\
\hline Total & 1.902 & 1 & 6 & 541 & 1.104 & 171 & 23 & 11 & 45 \\
\hline
\end{tabular}

Tableau synoptique des 1.902 chaînes anthroponymiques de la base de données PatRom qui s'ouvrent sur un article défini

\subsubsection{Aspect diachronique}

Une lecture non avertie du tableau ci-dessus pourrait suggérer l'idée que les chaînes anthroponymiques modifiées par un article défini sont typiques du $13^{\mathrm{e}}$ et encore plus du $14^{\mathrm{e}}$ siècle (541 occurrences, soit $28 \%$ du total de 1.902 , pour le $13^{\mathrm{e}}$ siècle et 1.104 occurrences ou $58 \%$ pour le $14^{\mathrm{e}}$ siècle). Une telle conclusion serait pourtant erronée, car ces nombres ne font que refléter des choix opérés au sein du projet PatRom : considérant que les $13^{\mathrm{e}} / 14^{\mathrm{e}}$ siècles représentaient une période charnière pour la formation du système anthroponymique roman, ces deux siècles ont été privilégiés lors de la sélection des sources à saisir.

\subsubsection{Aspect catégoriel}

Le deuxième critère d'évaluation concerne l'aspect catégoriel, c'est-à-dire la nature de l'élément suivant directement l'article défini, que l'on appellera pivot. Conformément aux trois grandes catégories onomastiques qui se dégagent sur la base du critère étymologique ${ }^{10}$, il peut s'agir soit d'un nom de personne originel, soit d'un lexème ou alors d'un nom de lieu originels (éventuellement devenus à leur tour nom de personne).

Le cas de loin le plus représenté concerne les pivots lexématiques (lexèmes ou anthroponymes d'origine délexicale, la différence entre les deux n'étant pas toujours aisée à faire). Considérant que tous les lexèmes pouvant occuper cette place ne jouaient pas le même rôle en anthroponymie, nous avons subdivisé cette catégorie en trois groupes : d'une part les titres ( $l i$ sires Forquez de Jurue), d'autre part les introducteurs (la dite Pedoge), qui sont tous les deux obligatoirement suivis d'un ou de plusieurs élément(s) anthroponymique(s) supplémentaire(s) (Forques et de Jurue; Pedoge), et nous avons appelé lexèmes stricto sensu ceux qui peuvent se suffire à eux-mêmes (comme dans l'Orfevre).

C'est ce dernier groupe qui occupe très clairement la première place : 1.515 chaînes anthroponymiques $(80 \%)$ présentent la structure : article + lexème stricto sensu (+ éventuellement X). En voici quelques exemples : la Tirefer (Paris a.1297), li Beigues (Troyes s.13), l'Orfevre (Ponthieu a.1311/1312), le Boçu savetier (Champagne a.1338), li Clerc de Rotechault (Besançon s.14), la Caille de Buncey (Châtillon-sur-Seine a.1430/1432) ou encore la Frisée (Beaune a.1470).

Avec 233 occurrences (12\%), les chaînes présentant la structure : article + titre $+\mathrm{X}$ sont relativement fréquentes aussi : li sires Forquez de Jurue (Metz a.1251), li segneur Boucquin Chielairon li maistre eschevin de Mets (Metz s.14), la dame de la Milloe (Paris a.1297).

\footnotetext{
${ }^{10}$ La catégorie numériquement faible des délocutifs (cf. Chambon 1990) n'est pas représentée dans notre corpus.
} 
Comparativement, les chaînes qui obéissent à la structure : article + introducteur $+\mathrm{X}$ sont bien moins fréquentes : on ne compte que 35 occurrences (2\%), comme la dite Pedoge (Poitiers a.1296), li diz Bernars Tronkiere (Paris s.13), li dicte dame Marieu Macqueriele (Cysoing a.1393), le dit Petit (Troyes a.1547) ou encore le nommé Bunel (Verson a.1790).

Si le gros des attestations concernent des chaînes anthroponymiques dont le premier (et éventuellement seul) élément après l'article défini est un lexème, 117 occurrences $(6 \%)$ associent le déterminant à un pivot nom de personne : la Guyomarde de Vile Dolz (Aunis a.1246), li Jordan Fraessens (Arras s.13), le Francois (Ponthieu a.1311/1312), la Germaine (Tours a.1314), ly Jehan de Chesseigne (Besançon a.1386).

Le cas des pivots toponymes (en l'occurrence, anthroponymes d'origine détoponymique), enfin, est tout à fait exceptionnel; seules deux chaînes anthroponymiques (moins de 1\%) sont constituées d'un article défini suivi d'un nom de lieu : la Biaroe (Niort a.1249/1271) et la Villeret (Châtillon-sur-Seine a.1430/1432). Dans le cas de la Biaroe, l'hypothèse détoponymique est suggérée par les parallèles Joh. de Biaroe (Niort a.1249/1271), Marce de Biaroe (Niort a.1249/1271) et Poquet de Biaroe (Niort a.1249/1271), tous les trois relevés dans la même source et contenant la préposition $d e$, qu'il est difficile d'interpréter autrement que comme un indicateur de provenance ("Johannes qui vient de Biaroe", "Marce qui vient de Biaroe", etc.) ${ }^{11}$. Pour ce qui est de la Villeret, relevé à Châtillon-sur-Seine (dans le nord de la Côte-d'Or) ${ }^{12}$, le nom de personne semble se rattacher au toponyme Villeret (nom d'une commune située dans l'est de l'Aube, attesté depuis 854 [Villa rivi], Boutiot/Socard 1874).

\subsubsection{Aspect syntaxique}

Après le critère catégoriel, notre intérêt se portera sur l'aspect syntaxique, c'est-à-dire sur la structure interne des chaînes anthroponymiques. Pour ce qui est des lexèmes stricto sensu - nous laisserons de côté les titres et les introducteurs, qui demandent par définition une expansion -, on compte un peu plus de chaînes simples (article + lexème : li Beigues ; 800 occurrences ou 53\%) que de chaînes complexes (article + lexème $+\mathrm{X}$ : le Boçu savetier ; 715 occurrences ou $47 \%$ ), ce qui ne paraît guère significatif.

En revanche, les noms de personnes sont précédés de l'article défini nettement plus souvent quand ils se présentent seuls (article + anthroponyme : la Germaine ; 95 occurrences ou $81 \%$ ) que quand ils sont suivis d'un autre élément (article + anthroponyme $+\mathrm{X}$ : ly Jehan de Chesseigne ; 22 occurrences ou 19\%). Cette dernière catégorie, peu représentée, tire sa raison d'être de la situation de communication: dans le cas de noms de baptême fréquents comme Jean, il peut aisément y avoir ambiguïté référentielle, de sorte que le scribe identifie le référent à travers la corrélation article défini + anthroponyme + expansion (cf. Gary-Prieur 1994, 105-117). Pour ce qui est du type la Germaine, majoritaire, l'explication de sa fréquence échappe à la syntaxe ; nous y reviendrons sous 2.2.4.4.

Les deux chaînes anthroponymiques où l'article détermine un nom de lieu originel, enfin, ne contiennent pas d'expansion (la Biaroe et la Villeret).

\subsubsection{Aspect diatopique}

2.2.4.1. Nous avons vu que la diachronique n'est pas pertinente pour l'interprétation de nos données. Parmi les aspects diasystématiques que distingue la linguistique variationnelle, il convient toutefois de s'interroger sur l'éventuel pouvoir explicatif du second critère utilisable sans grande difficulté pour les états anciens de la langue: le critère diatopique. Nous commencerons par comparer le nombre total des chaînes anthroponymiques disponibles dans la base de données pour les huit domaines dialectaux délimités par PatRom avec le nombre respectif des chaînes anthroponymiques débutant par un article défini :

\footnotetext{
${ }^{11}$ Ce qui, en l'état actuel de nos connaissances, fragilise l'intreprétation détoponymique, c'est que nous n'avons pas identifié le nom de lieu éponyme. Mais la base de données PatRom ne contient aucune autre occurrence de Biaroe, ce qui rend la cohérence des quatre occurrences particulièrement pertinente.

${ }^{12}$ La base de données PatRom ne contient aucune autre occurrence de Villeret.
} 


\begin{tabular}{|l|r|r|}
\hline & Occurrences & Occurrences commençant par un article défini \\
\hline français central & 75.380 & 150 \\
\hline haut-breton & 14.799 & 75 \\
\hline poitevin & 9.528 & 96 \\
\hline normand & 11.231 & 74 \\
\hline picard & 29.673 & 841 \\
\hline lorrain & 18.916 & 249 \\
\hline champenois & 4.606 & 100 \\
\hline bourguignon et franc-comtois & 8.331 & 317 \\
\hline Total & $\mathbf{1 7 . 2 4 6 4}$ & $\mathbf{1 . 9 0 2}$ \\
\hline
\end{tabular}

En raison de l'important écart numérique entre les deux plages de données, une représentation graphique reposant sur les pourcentages respectifs plutôt que sur les chiffres absolus sera néanmoins plus parlante :
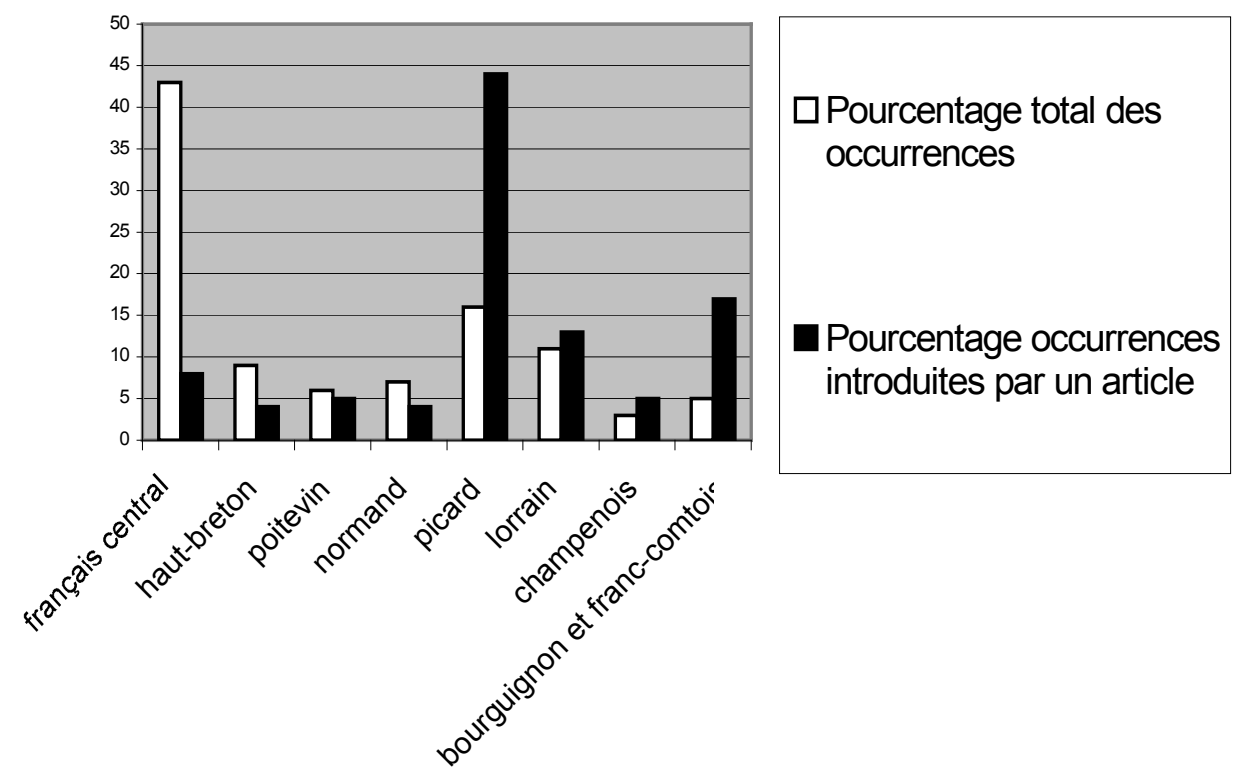

Pour cinq des huit domaines linguistiques, les pourcentages des occurrences totales et ceux des occurrences introduites par un article défini sont assez comparables : respectivement 9 et $4 \%$ pour le haut-breton, 6 et $5 \%$ pour le poitevin, 7 et $4 \%$ pour le normand, 11 et $13 \%$ pour le lorrain et 3 et $5 \%$ pour le champenois ${ }^{13}$. En revanche, les données du français central, celles du picard et celles du bourguignon et du franc-comtois appellent des commentaires.

2.2.4.2. On commencera par le français central. Si $43 \%$ des occurrences formant la base de données PatRom appartiennent au français central, $8 \%$ de celles avec article seulement ressortissent à ce domaine. Cela constitue un premier résultat exploitable : le domaine du français central, dont les données proviennent essentiellement de sources parisiennes (rôles de taille de 1292, 1296, 1297, 1298, 1299, 1300, 1313, 1421, 1423, 1438 et plusieurs cartulaires, réunissant divers documents des $12^{\mathrm{e}}$ au $14^{\mathrm{e}}$ siècles), est relativement pauvre en chaînes anthroponymiques déterminées par un article. Et, dans la mesure où c'est «la langue de la capitale administrative, intellectuelle et démographique, scripta de la cour et de la chancellerie royale, qui est en quelque sorte l'ancêtre du français standard moderne»»

\footnotetext{
${ }^{13}$ Compte tenu des hasards inévitables de l'échantillonnage, on considérera que ces légers écarts ne sont pas significatifs.
} 
(Buchi/Glessgen 2001, 76), on tient peut-être là une des raisons du caractère périphérique du nom de personne modifié en français moderne.

2.2.4.3. À l'inverse, le picard, bien que beaucoup moins représenté dans la base de données (16\% des occurrences), concentre près de la moitié $(44 \%)$ des chaînes anthroponymiques introduites par l'article. Cependant, à y regarder de plus près, cette concentration ne concerne pas l'ensemble des sources du domaine picard, mais seulement le Nécrologe de la confrérie des jongleurs et des bourgeois d'Arras (a.1194-1361) : sur les 841 occurrences picardes, $580(69 \%)$ proviennent de cette source, ce qui est considérable. Et le nécrologe d'Arras présente la particularité de nommer assez communément les personnes citées non pas dans l'ordre nom de baptême + surnom, mais dans l'ordre inverse surnom + nom de baptême (li Jais Jehans, le Jongleresse Catherine) ${ }^{14}$, coutume qui rappelle notre usage moderne pour les bibliographies. On est donc là en présence d'une manifestation inversée d'un phénomène bien connu, mais exclu de notre corpus $(\rightarrow 2.1$.).

2.2.4.4. Reste le domaine bourguignon et franc-comtois, qui, quoique ne fournissant que $5 \%$ des chaînes anthroponymiques de la base, totalise $17 \%$ de celles qui s'ouvrent sur un article défini. Ce déséquilibre s'explique, au moins partiellement, par un nombre très élevé de noms de baptême déterminés par un article défini (la Nichole dou Pavoillon [Salins a.1275], li Francoise [Besançon a.1386], la Rolande [Beaune a.1442]). Lorsque l'on ne considère que les chaînes anthroponymiques sans expansion (type li Francoise, la Rolande), la prépondérance du domaine de l'est oïlique apparaît avec encore plus de netteté : le graphique ci-dessous montre très clairement que, comparativement au nombre d'occurrences disponibles pour chaque domaine linguistique, c'est la Bourgogne et la Franche-Comté qui capitalisent de loin le plus de chaînes anthroponymiques constituées d'un article défini et d'un nom de baptême :

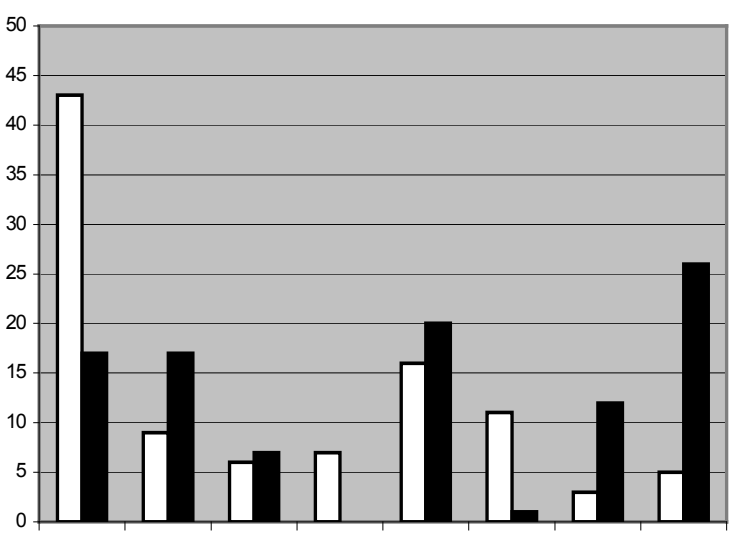

$\square$ Pourcentage total des occurrences

Pourcentage des occurrences formées d'un article et d'un nom de personne nu

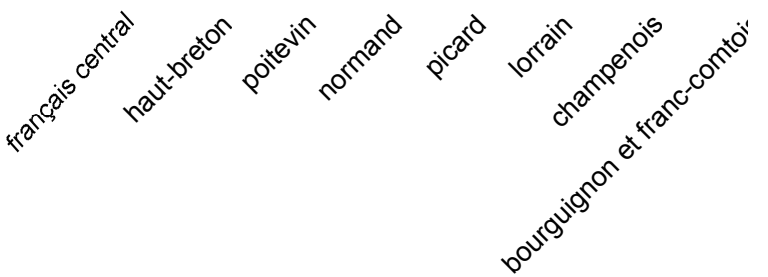

Il s'agit donc là d'un diatopisme oriental évident, auquel fait écho, à l'époque contemporaine, le particularisme syntaxique du français populaire - attesté notamment, mais pas exclusivement, dans l'Est de la France - qui consiste à faire précéder la mention des prénoms de

\footnotetext{
${ }^{14}$ Berger souligne «les habitudes si déroutantes des clercs de la confrérie. Dès 1196, il leur arrive de rejeter le prénom après le sur-nom. Cette manière de faire se généralise presque aussitôt, non sans souffrir quelques exceptions chaque année jusqu'aux environs de 1300 ; elle devient ensuite la règle pour aboutir à des inscriptions comme celles-ci : Grumeliers Robers li, Paris Jakemes de. Une telle pratique, dont je ne connais aucun autre exemple, avait quelque chose de révolutionnaire [...]» (Berger 1970, 57).
} 
l'article défini (la Bernadette et l'Hubert) ${ }^{15}$. Si notre corpus permet d'observer la même tendance en Champagne, on est étonné par la quasi-absence du phénomène dans le domaine lorrain. À moins de supposer une erreur dans notre manière d'exploiter les données, il faut donc supposer que cette particularité syntaxique est d'introduction plus récente en Lorraine.

2.2.4.5. On terminera par une remarque rapide sur la situation en Normandie. On a vu sous 2.2.4.1. que le domaine normand ne présente aucune concentration notable de chaînes anthroponymiques s'ouvrant sur un article défini. Ce fait pourra paraître surprenant, car la Normandie est la région de France où l'on relève la plus forte densité de noms de famille à article agglutiné du genre Labbé, Lacaille, Leblanc, Leboucher ou Levillain ${ }^{16}$. Il faut en conclure que ce type patronymique tire son origine non pas de chaînes anthroponymiques entières, mais de surnoms occupant en général la seconde position à l'intérieur de ces chaînes, comme dans Aelicia la Borgeise (Saint-Vigor a.1292) ou Bernart le Mire (Eu a.1307).

\subsubsection{Aspect morphologique}

Le dernier critère auquel on peut faire recours pour cerner la spécificité des chaînes anthroponymiques modifiées est d'ordre morphologique. Il ne concerne que les chaînes qui font référence à un porteur du sexe féminin et, à l'intérieur de cette catégorie, uniquement celles dont le pivot est un anthroponyme.

Notre base de données fournit 64 chaînes anthroponymiques présentant un article défini féminin suivi d'un nom de personne féminin. Pour plusieurs d'entre ces derniers, on relève, chaque fois dans la même source, des formes masculines sur lesquelles ils ont visiblement été formés : la Bernardote (Aunis a.1246) voisine avec Martin Bernardot (Aunis a.1246), la Racaude (Niort a.1249/1271) avec Racaus (Niort a.1249/1271), la Droinesse (Champagne a.1295) avec Droins Patins (Champagne a.1295), la Rinaude (Salins-les-Bains a.1276) avec Rinaut au Crot de Chimin (Salins-les-Bains a.1276) et la Viennotte (Beaune a.1470) avec Viennot le Bon (Beaune a.1470). En résumé, on a donc ${ }^{17}$ :

$\begin{array}{ll}\text { (Martin) Bernardot } & \rightarrow \text { la Bernardote } \\ \text { Racaus } & \rightarrow \text { la Racaude } \\ \text { Droins (Patins) } & \rightarrow \text { la Droinesse } \\ \text { Rinaut (au Crot de Chimin) } & \rightarrow \text { la Rinaude } \\ \text { Viennot (le Bon) } & \rightarrow \text { la Viennotte }\end{array}$

Tandis que les bases masculines sont dépourvues de l'article, mais présentent en général une expansion (Martin, Patins, etc.), leurs correspondants féminins sont précédés de l'article, mais présentent comme toute «expansion» une marque morphologique du féminin $(-e,-d e$, -esse, -te). L'article contribue donc, comme les expansions des chaînes masculines, à lever l'ambiguité référentielle. On peut dire avec M.-N. Gary-Prieur (1994, 104 et note 3) que «la

\footnotetext{
${ }^{15}$ Cf. Lanher/Litaize 1990 [Lorraine] s.v. la, le; Boisgontier 1991 [Aquitaine] s.v. le, la («usage d'origine dialectale répandu dans tous les pays gascons et garonnais, inconnu dans la région pyrénéenne») ; Robez-Ferraris 1995 [Jura] s.v. la, le («ce tour semble largement répandu dans les milieux ruraux et dans certaines régions»); Fréchet 1997 [Drôme] s.v. le, la, les ; Fréchet/Martin 1998 [Ain] s.v. le, la ; Benoît/Michel 2001 [Metz] s.v. le, la, les («régionalisme grammatical de grande extension»). Contrairement au français (vulgaire) général (cf. Grevisse/Goosse 1993, 339), cet usage n'implique aucune nuance de dédain ou de mépris.

${ }^{16}$ «Plus caractéristique la présence ou l'absence de l'article devant un nom de profession (Mercier-Lemercier), soit devant un sobriquet appartenant à l'une des catégories suivantes : noms de dignité (Roy-Leroy), nom commun employé par métaphore (Bœuf-Lebœuf), adjectif substantivé pour désigner une particularité d'ordre physique ou moral (Roux-Leroux). [...] Bien qu'aucune démarcation nette ne puisse être établie en l'absence de matériaux suffisants, on peut dire que l'article est absent dans le Sud-Est, du Languedoc à la Suisse romande inclus ; les formes avec et sans article se rencontrent au Sud-Ouest, dans le Massif Central, le Centre et l'Est de la langue d'oïl ; le Nord-Ouest et le Nord, spécialement la Normandie et le Hainaut, n'offrent guère que des noms précédés de l'article agglutiné.» (Dauzat 1977, 303). Cette particularité de la Normandie est confirmée par les recherches réalisées dans le cadre du projet PatRom.

${ }^{17}$ Il est à remarquer qu'aucune forme féminine n'a été formée à partir d'un nom de baptême très fréquent (*la Jehanne, *la Martine) : on peut penser qu'avec de tels noms le risque d'homonymie aurait été trop important.
} 
forme le $N p$ correspond à un repérage anaphorique, l'article présupposant linguistiquement l'unicité du référent [...] : l'article anaphorique renvoie à un 'référent saillant' dans la mémoire des protagonistes de l'énoncé».

En ce qui concerne les huit chaînes formées de l'article défini féminin, d'un nom de baptême féminin et d'une expansion, nous n'avons pas relevé de correspondants masculins assurés. Trois en revanche présentent des noms de baptême bien représentés dans la base de données : la Fremine pastaiere (Paris a.1297), la Jehanne de Grantson ma damoiselle (Besançon a.1400) et la Nichole dou Pavoillon (Salins-les-Bains a.1275). Dans ce type de chaînes anthroponymiques, c'est donc l'expansion qui joue le rôle d'individualisation que ne peut remplir à elle seule la féminisation.

On notera que cet usage médiéval s'est en partie perpétué en français moderne et contemporain, certes sans morphème féminisateur ; il est critiqué par les grammairiens : «le peuple se sert de l'article féminin pour désigner la femme (la Durand), tournure qui est du dernier vulgaire» (Dauzat 1977, 364).

\subsubsection{Tentative d'explication unifiée}

2.2.6.1. Les critères interrogés tout au long de cette interprétation des 1.902 chaînes anthroponymiques débutant par un article défini que présente la base de données PatRom nous permettent d'affirmer ce qui suit :

1. Le système anthroponymique français connaît des chaînes anthroponymiques modifiées par un article défini depuis au moins le $11^{\mathrm{e}}$ siècle, sans qu'aucune période de son histoire ne se recommande comme particulièrement typique du phénomène.

2. Le pivot des chaînes anthroponymiques françaises modifiées par un article défini est en règle générale un lexème, ou plus précisément, dans la majorité des cas, un nom propre de personne dont l'étymon est un lexème (la différence entre les deux, radicale en théorie, étant souvent difficile à faire dans la pratique).

3. Certaines chaînes anthroponymiques françaises modifiées par un article défini comportent un élément autre que l'article et l'unité linguistique qu'il détermine directement. Cela est obligatoire quand le pivot est un titre ou un introducteur, minoritaire dans le cas des lexèmes stricto sensu et des anthroponymes, non attesté dans le celui des toponymes.

4. Du point de vue diatopique, deux particularités se dégagent : a) le français parisien et central connaît nettement moins de chaînes anthroponymiques modifiées par un article défini que les variétés géographiques périphériques; b) une partie de l'est du domaine français (bourguignon et franc-comtois) concentre un nombre particulièrement élevé de chaînes anthroponymiques modifiées par un article défini dont le pivot est un anthroponyme.

5. Quand le pivot d'une chaîne anthroponymique française modifiée par un article défini est constitué d'un anthroponyme féminin, ce dernier représente souvent un nom de personne masculin auquel on a ajouté une marque de féminin.

2.2.6.2. Sans mettre en doute l'intérêt d'une telle vision particularisante, on essaiera à présent de proposer une interprétation unifiée des 1.902 chaînes anthroponymiques introduites par l'article défini que comporte notre corpus. Pour cerner le dénominateur commun des différents cas de figure distingués ci-dessus, la notion de description définie, introduite par B. Russell et précisée par G. Kleiber, se révèle, nous semble-t-il, particulièrement féconde. Kleiber (1981, 173-174) appelle description définie tout syntagme nominal qui commence par un article défini singulier et qui renvoie à un particulier. Les chaînes anthroponymiques qui s'ouvrent sur un article défini représentent donc un type spécifique de descriptions définies, leur particularité consistant à comporter obligatoirement un nom propre de personne : li Beigues, le Boçu savetier, li sires Forquez de Jurue, le dit Petit, le Francois, ly Jehan de Chesseigne et la Biaroe répondent tous à la définition de la description définie.

C'est le cadre de la théorie de la description définie qui permet d'assigner un rôle unifié à l'article, à l'interface entre l'ambiguïté référentielle de tout nom propre de personne, qui 
peut être porté par un nombre illimité d'individus, et la situation extra-linguistique, qui sélectionne un seul porteur à l'intérieur de cet ensemble théorique, celui que le scribe entend désigner de façon univoque : «l'article défini a pour effet de limiter cette classe à un et un seul individu» (Kleiber 1981, 173) ${ }^{18}$.

Aucune des catégories délimitées ci-dessus ne contraint, certes, le locuteur (en l'occurrence, plutôt le scripteur) à l'usage de l'article. En effet, on relève, pour chaque type modifié, des parallèles structurels non modifiés, même si certains d'entre ces derniers, comme dans le cas de l'introducteur dit, sont très minoritaires :

$\begin{array}{lll}\text { li Beigues } & - & \text { Roussele (Ponthieu a.1311/1312) } \\ \text { le Boçu savetier } & - & \text { Petit de Chalons (Provins a.1285) } \\ \text { li sires Forquez de Jurue } & - & \text { sire Androwin Roucel (Metz a.1505) } \\ \text { le dit Petit } & - & \text { dit Girart Meguet (Salins a.1253) } \\ \text { le Francois } & - & \text { Francois (Artois env. a.1300) } \\ \text { ly Jehan de Chesseigne } & - & \text { Jehan de Lusigny (Troyes a.1429) } \\ \text { la Biaroe } & - & \text { Besançon Bourreler (Beaune a.1423) }\end{array}$

Ainsi, quand le scribe se sert de l'article défini pour nommer un individu, on en conclura que l'unicité référentielle lui paraissait problématique, que la raison en soit d'ordre extralinguistique (désambiguïsation référentielle) ou linguistique (désambiguïsation co[n]textuelle) : " On peut observer que le Npr modifié dénominatif est le type qui apparaît lorsque la modification du NPr semble due à des facteurs relevant de la situation d'énonciation. Normalement, il trahit alors l'incapacité du locuteur à faire un emploi référentiel du NPr non modifié »" (Jonasson 1994, 183).

2.2.6.3. Cette analyse vaut pour une bonne partie des 1.902 chaînes anthroponymiques modifiées du corpus PatRom. En tout état de cause, elle pourra être invoquée dans l'explication de l'apparition de l'ensemble des constructions analysées ici. Par la suite, toutefois, certains de ces tours se sont imposés avec une certaine régularité dans un point particulier du diasystème français.

\section{De la description définie au nom de famille}

3.1. Nous terminerons notre étude par un rapide excursus sur les traces éventuelles de la prédétermination de certaines chaînes anthroponymiques telle qu'elle apparaît dans les documents médiévaux, phénomène éminemment discursif, dans le stock anthroponymique français contemporain, qui, lui, relève du système linguistique. Nous examinerons le devenir (maintien ou disparition) de l'article défini lors de la fixation des noms de famille, espérant obtenir ainsi des renseignements sur le statut plus ou moins figé de l'article lors du passage du nom de personne individuel au nom de famille héréditaire ${ }^{19}$. À ce titre, notre dessein participe de l'entreprise amorcée par l'une d'entre nous pour la lexicalisation déonomastique des noms propres à article intégré (Le Corbusier > lecorbuserie n.f. "bâtiment dans le style de Le Corbusier"; cf. Buchi 2002).

\footnotetext{
${ }^{18}$ Un contexte un peu plus large permettra de mieux situer cette assertion : «un substantif individuant présuppose l'existence d'une classe d'individus. Or, l'article défini a pour effet de limiter cette classe à un et un seul individu. Nous sommes donc en face de la situation suivante : d'une part, le substantif présuppose plusieurs individus possibles, d'autre part, l'article défini dit qu'il n'y en a qu'un. Pour que cette information ne soit pas contradictoire, il faut obligatoirement des indices référentiels qui permettent, par la restriction qu'ils apportent, d'éviter la contradiction entre les indications apportées par l'article défini et celles du substantif individuant» (Kleiber 1981, 173).

${ }^{19}$ Pour les époques de fixation des noms de famille, échelonnées par régions, cf. Dauzat 1977, 38-48 et Bourin $1990-2002$.
} 
3.2. Un premier stade de la fixation progressive concerne l'agglutination de l'article au pivot $^{20}$. Cette dernière est attestée dès la fin du $12^{\mathrm{e}}$ siècle (Lafranceise [Normandie 1195]), mais elle est relativement peu fréquente et reste confinée aux pivots lexèmes stricto sensu (Lepage [Verson a.1247], Laforest paticher [Louviers a.1597]) et aux pivots introducteurs (lidit Passavant [Besançon s.14], lenommé Laroche [Combs a.1791]). En tout état de cause, vu le caractère fluctuant de la séparation des mots pratiquée par les scribes médiévaux ${ }^{21}$, le pouvoir démonstratif de ce phénomène purement graphique reste quelque peu limité.

3.3. Un regard sur la situation moderne et contemporaine, postérieure au processus de patronymisation, sera plus éclairant. En effet, l'existence de nombreux noms de famille composés étymologiquement d'un lexème stricto sensu et d'un article défini intégré, soudé ou non (Le Petit/Lepetit) ${ }^{22}$, atteste d'un état avancé de figement du déterminant au moment du passage du nom de personne individuel au nom de personne héréditaire ${ }^{23}$. Le témoignage de ce type de formations est toutefois peu concluant en soi, car rien ne prouve que les noms de famille comme Lepetit soient issus de la fixation de chaînes prédéterminées du genre Le Petit Perrin (Beaune a.1400). Au contraire, étant donné la suprématie numérique des chaînes où l'article détermine la seule expansion (ainsi Jehan le Petit [Paris a.1297]) ${ }^{24}$, on aura tendance à affirmer que ces patronymes reposent le plus souvent sur ce dernier type de mentions.

3.4. Cette incertitude quant à l'origine syntaxique des patronymes à article intégré frappe l'ensemble des pivots lexématiques. À titre d'exemple, nous avons tenté de lever l'ambiguïté pour la formule le dit $X^{25}$, dont l'exploitation statistique paraissait maîtrisable. Ce tour s'est fixé dans le stock patronymique français sous la forme du nom de famille $\mathrm{Ledit}^{26}$, typique de l'Est de la France et attesté tant à la fin du $19^{\mathrm{e}}$ siècle (Doubs, Haut-Rhin, Territoire de Belfort, Vosges, cf. Fordant 1999) qu'à l'époque contemporaine (29 abonnés au téléphone, avec une concentration notable dans l'Aube et dans le Territoire de Belfort ${ }^{27}$ ). L'ancêtre médiéval de ce patronyme ne peut qu'être constitué d'une chaîne anthroponymique modifiée, car au vu du corpus PatRom, la séquence le + dit n'a d'existence qu'en ouverture de chaîne anthroponymique, à l'exclusion de tout usage en position interne $\left({ }^{*} X\right.$ le dit $\left.Y\right):$ la totalité des dix-huit occurrences de le dit (le dit Maussacre [Poitiers a.1296], le dit Huguenot [Boulognesur-Mer a.1415/1416], le dit Petit [Troyes a.1547], etc.) et l'ensemble des vingt-et-une occurrences de ledit (ledit Perreaul [Bourgogne a.1374/1375], ledit Estevenin Chevriat [Besançon a.1384/1387], ledit Jehan le Rouziés [Metz a.1440], etc.) présentent en effet le tour en début de chaîne.

Certes, il s'agit là d'un témoignage isolé et très particulier, et il est évident que des études analogues à partir de pivots lexématiques choisis seront nécessaires pour apprécier

\footnotetext{
${ }^{20} \mathrm{Cf}$. Germain 2002 pour cette question. L'auteur met en évidence l'importance décisive du nombre de syllabes du nom de personne pour le processus de soudure ou non.

${ }^{21}$ «Old French scribes were less conscious than we are of the limits of those language units we call words ; consequently, groups of words are often run together in manuscripts» (Foulet/Speer 1979, 58-59).

${ }_{22}$ Cela concerne non seulement la Normandie, région où l'on observe le maximum d'intensité de ce type de formations, mais l'ensemble du domaine oillique $(\rightarrow 2.2 .4 .5$. et note 18$)$.

${ }^{23}$ Ce postulat n'empêche pas, comme nous le suggère Jean-Pierre Chambon, d'envisager l'article en synchronie comme un translatif marquant l'anthroponymisation (cf. Lemaréchal 1989, 27-28 ; 36-38; 46-48 et passim).

${ }^{24}$ Nous ne sommes pas en mesure de quantifier cette suprématie numérique, mais un maniement régulier des sources anthroponymiques médiévales nous en donne l'intime conviction.

${ }^{25}$ Pour une typologie des noms de personne comportant l'élément dit, cf. Vincent 1958.

${ }^{26} \mathrm{Il}$ y a visiblement eu réanalyse au cours des siècles, car le tour servait au début à la simple reprise d'un anthroponyme déjà mentionné, comme dans Osennete, borjoise dou Nuef Chastel (Neufchâteau a.1265), repris quelques lignes plus bas par : aprés le decés la-dite Osannete (Neufchâteau a.1265 ; cf. Lanher 1975, 112-113). Au départ, le tour ne pouvait donc pas avoir d'existence indépendante.

${ }^{27} \mathrm{La}$ répartition géographique des porteurs des noms de famille contemporains a été extraite du cédérom de France Telecom (1998). Nos remerciements s'adressent à Carole Champy (ATILF, Nancy), qui a bien voulu se charger de cette partie du travail.
} 
l'ampleur du phénomène. Il n'en reste pas moins que le cas de ledit est éloquent, car il nous montre qu'à un moment donné (qui ne coïncidera pas, en règle générale, avec l'instant représenté par le document historique), dans la conscience des locuteurs, le modificateur originel a basculé dans le champ du nom propre. Cette absorption par le nom propre de ce qui, au départ, constituait un simple déterminant nous semblait digne d'être relevée.

\section{Conclusion}

Tout au long de cette recherche, le recours aux notions développées dans le cadre de la théorie du nom propre pour l'analyse d'un corpus anthroponymique historique s'est montré fécond ; les références aux travaux de N. Flaux, M.-N. Gary-Prieur, K. Jonasson, G. Kleiber et M. Noailly en témoignent amplement.

Inversement, nous espérons avoir pu montrer que l'approche historique était capable d'apporter sa pierre à l'analyse du nom propre modifié, et cela dans deux domaines. En premier lieu, l'exploitation d'un vaste corpus original de chaines anthroponymiques essentiellement médiévales apporte un minimum de profondeur historique à notre connaissance du système anthroponymique français. Un second apport, encore réduit mais sans doute appelé à s'amplifier, semble se dégager de l'étude de la fixation patronymique de l'article (Ledit).

En conclusion, le dialogue entre anthroponymie historique et théorie du nom propre est désormais amorcé ; il s'agira bien sûr de le poursuivre et de l'approfondir.

\section{Bibliographie}

Benoît (Michèle)/Michel (Claude), 2001. Le Parler de Metz et du Pays Messin, Metz, Serpenoise.

Berger (Roger), 1970. Le Nécrologe de la confrérie des jongleurs et des bourgeois d'Arras (1194-1361), vol. 2 : Introduction, Arras, Mémoires de la Commission Départementale des Monuments historiques du Pas-de-Calais.

Boisgontier (Jacques), 1991. Dictionnaire du français régional des Pays Aquitains, Paris, Bonneton.

Bourin (Monique), 1990-2002. Genèse médiévale de l'anthroponymie moderne, 5 vol., Tours, Publications de l'Université de Tours.

Boutiot (Théophile)/Socard (Émile), 1874. Dictionnaire topographique du département de l'Aube, Paris, Imprimerie Nationale.

Buchi (Éva), 2001. "La méthodologie de l'étymologie des noms de famille (domaine français et galloroman)", Rivista Italiana di Onomastica 7, 105-127.

Buchi (Éva), 2002. "Réflexions sur l'apport de la déonomastique pour la théorie du nom propre : le cas des éponymes à article intégré (domaine roman)", in: Kremer (Dieter) (éd.), Onomastik. Akten des 18. Internationalen Kongresses für Namenforschung (Trier, 12.-17. April 1993), Tübingen, Niemeyer, vol. V, 171-188.

Buchi (Éva)/Glessgen (Martin-Dietrich), 2001. "Variétés locales et suprarégionales dans la genèse des langues romanes standard", Mémoires de la Société de Linguistique de Paris $11,65-86$.

Cano (Ana Maria)/Germain (Jean)/Kremer (Dieter) (éd.), 2004-. Dictionnaire historique de l'anthroponymie romane (PatRom), 6 vol., Tübingen, Niemeyer.

Chambon (Jean-Pierre), 1988. Compte rendu de Le Nom propre, Langages 66 (1982), Nouvelle Revue d'Onomastique 11/12, 177.

Chambon (Jean-Pierre), 1990. "Une catégorie souvent négligée de noms de personne : les délocutifs. Quelques problèmes de reconnaissance et de classification (domaine français)", in : Kremer (Dieter) (éd.), Dictionnaire historique des noms de famille romans. Actes du $I^{\text {er }}$ Colloque (Trèves, 10-13 décembre 1987), Tübingen, Niemeyer, 128-137. 
Chambon (Jean-Pierre), 1996. "Méthodes en anthroponymie historique : cinq noms de famille bas-auvergnats (Chassa[i]gnon, Vorilhon, Torrilhon, Fayon, Chandezon)", Rivista Italiana di Onomastica 2, 263-285.

Chambon (Jean-Pierre), 1998. "Méthodes en anthroponynie historique : à propos du nom de famille occitan Dental et congénères et de l'article DENTĀLE du Dictionnaire historique de l'anthroponymie romane", in: Cano González (Ana María)/Villa Basalo (Susana) (éd.), Dictionnaire historique des noms de famille romans [IX]. Actas del IX Coloquio (Uviéu/Oviedo, 26-29 de octubre 1995), Tübingen, Niemeyer, 65-81.

Chambon (Jean-Pierre), 2004. "L'onomastique du censier interpolé ( ca 946) dans la charte de fondation du monastère auvergnat de Sauxillanges", Revue de linguistique romane 68, 105180.

Dauzat (Albert), $1977^{3}\left[1942^{1}\right]$. Traité d'anthroponymie française. Les noms de famille en France, Paris, Guénégaud.

Flaux (Nelly), 1995. "La catégorisation du nom propre", in: Noailly (Michèle) (éd.), Nom propre et nomination. Actes du colloque de Brest (21-24 avril 1994), Toulouse, Presses de l'Université de Toulouse-Le Mirail, 63-73.

Fordant (Laurent), 1999. Tous les noms de famille et leur localisation en 1900, Paris, Archives \& Culture.

Foulet (Alfred)/Speer (Mary Blakely), 1979. On Editing Old French Texts, Lawrence, Regents Press of Kansas.

France Telecom, 1998. Le Disque Télécom. L'annuaire de France Télécom sur CD ROM, Paris, France Telecom.

Fréchet (Claudine), 1997. Dictionnaire du parler de la Drôme, Valence, E \& R.

Fréchet (Claudine)/Martin (Jean-Baptiste), 1998. Dictionnaire du français régional de l'Ain, Paris, Bonneton.

Gary-Prieur (Marie-Noëlle), 1994. Grammaire du nom propre, Paris, P.U.F.

Germain (Jean), 2002. "Du surnom au nom de famille. La fixation formelle des noms de famille en domaine d'oïl à partir de l'exemple de Namur (Wallonie)", in : Boullón Agrelo (Ana Isabel) (éd.), Actas do XX Congreso Internacional de Ciencias Onomásticas (Santiago de Compostela, 20-25 setembro 1999) [cédérom], A Coruña, Instituto da Lingua Galega, 1409-1414.

Grevisse (Maurice)/Goosse (André), $1993^{13}\left[1936^{1}\right]$. Le bon usage : grammaire française, Paris, Duculot.

Jonasson (Kerstin), 1994. Le Nom propre. Constructions et interprétations, Louvain-laNeuve, Duculot.

Kleiber (Georges), 1981. Problèmes de référence: descriptions définies et noms propres, Metz, Centre d'Analyses Syntaxiques de l'Université de Metz.

Kleiber (Georges), 1992. "Quand le nom propre prend article : le cas des noms propres métonymiques", French Language Studies 2, 185-205.

Kremer (Dieter) (coord.), 1997. Dictionnaire historique de l'anthroponymie romane (PatRom). Présentation d'un projet, Tübingen, Niemeyer.

Lanher (Jean), 1975. Chartes en langue française antérieures à 1271 conservées dans le département des Vosges, Paris, Éditions du CNRS.

Lanher (Jean)/Litaize (Alain), 1990. Dictionnaire du français régional de Lorraine, Paris, Bonneton.

Lemaréchal (Alain), 1989. Les Parties du discours. Sémantique et syntaxe, Paris, Presses Universitaires de France.

Noailly (Michèle), 1999. "La querelle des noms propres", Modèles linguistiques 39, 107-112.

Robez-Ferraris (Jacqueline), 1995. Particularités du français parlé dans la région de Morez, Haut-Jura, Grenoble, ELLUG. 
Vincent (Auguste), 1958. "L'emploi de dit dans les noms de personnes", Bulletin de la Commission Royale de Toponymie et dialectologie 32, 195-216.

Wirth (Aude), 2002. Prolégomènes à un dictionnaire des noms de famille lorrains, 2 vol. (167 + 73 pages), DEA Université Marc Bloch de Strasbourg, sous la direction de MartinDietrich Glessgen (soutenance : 26/6/2002).

Wirth (Aude), 2003. Eléments d'anthroponymie lorraine. Application de la méthodologie PatRom à une sélection de noms de famille, Dijon, Association Bourguignonne d'Études Linguistiques et Littéraires. 